Original Research Paper

\title{
Development and Optimization of Plant Extract Loaded Nanoemulsion Mixtures for the Treatment of Inflammatory Disorder
}

\author{
${ }^{1}$ Mukesh Gohel, ${ }^{2}$ Tejal Soni, ${ }^{3}$ Lal Hingorani, ${ }^{1}$ Asha Patel and ${ }^{2}$ Nayan Patel \\ ${ }^{1}$ Anand Pharmacy College, Anand, 388001, Gujarat, India \\ ${ }^{2}$ Faculty of Pharmacy, Dharmsinh Desai University, Nadiad, 387001 Gujarat, India \\ ${ }^{3}$ Pharmanza Herbal Pvt Ltd. Dharmaj, Gujarat, India
}

Article history

Received: 06-11-2014

Revised: 28-11-2014

Accepted: 29-12-2014

Corresponding Author:

Asha Patel,

Anand Pharmacy College,

Email: patelasha1405@gmail.com
Anand, 388001, Gujarat, India

\begin{abstract}
In current study, a nanoemulsion as drug delivery system (NE) of spray dried Boswellia Serrata Extract (BSE) was developed and characterized to compare it in vitro/Ex vivo diffusion with reference formulation. Pseudo-ternary phase diagrams were constructed by phase diagram by aqueous titration method, of which the evaluation method was improved for solubility, Droplet size and transmittance. The formulation of BSE-loaded NE was optimized by a Simplex lattice mixture experimental design. The optimized nanoemulsion formulation, loading with $199.8 \mathrm{mg} \mathrm{g}^{-1}$ BSE total boswellic acids, consisted of Isopropyl Myristate, Tween 80 and Ethanol $(2: 8: 10, \mathrm{w} / \mathrm{w})$ and it remained stable after storing at $40^{\circ} \mathrm{C}, 25^{\circ} \mathrm{C}, 4^{\circ} \mathrm{C}$ for at least 6 months. When diluted with water, the NE droplet size was 11.25 $\mathrm{nm}$ and the zeta potential was $0.223 \mathrm{mV}$. The spherical droplet shape was confirmed by TEM analysis. Ex vivo skin permeation and in vivo antiinflammatory study were conducted. About 3.25 fold increase in flux was seen in case of nanoemulsion, nanogel showed 1.45 fold increase in flux as compared to carbopol gel with highest enhancement ratio 4.57 and 1.59 respectively. Our study illustrated potential of developed nanoemulsion as possible alternative to traditional topical formulations.
\end{abstract}

Keywords: Boswellia Serrata Extract, Nanoemulsion, Simplex Lattice Mixture Design

\section{Introduction}

Globally there is renewed interest in drugs derived from herbal sources due to its promising beneficial therapeutic effects and better tolerance with no side effects. Currently, pharmacological and clinical studies indicate that gum resin of Boswellia serrata $[B$. serratta] contains six types of Boswellic Acids (BAs) as active herbal principle which play significant role in treatment of inflammatory disorders by inhibiting 5-Lipoxygenase (5-LO) (Gerbeth et al., 2011). Herbal drugs have therapeutic potential which shall be explored through some value added new drug delivery system. Nanoemulsions are optically isotropic, transparent, thermodynamically stable systems of oil, surfactants and water with miniscule droplet size, high solubilization capacity for lipophilic drugs (Azeem et al., 2009), ease of preparation, enhanced biocompatibility and size of emulsion droplets influence its target distribution (Ajazuddin, 2010) thereby improve the penetration of drug into skin (Mayank et al., 2011).
Many plant extracts have been incorporated in nanoemulsion for transdermal delivery, but boswelllic acids have not been evaluated into nanoemulsion vehicle. However novel Boswellic acids nanoparticle have been developed for prostate cancer cell death (Uthaman et al., 2012) and 3 Acetyl-11 keto-B boswellic acid loaded polymeric nanomicelles significantly enhanced topical antiinflammatory and anti-arthritic activity (Goel et al., 2010).

In a nutshell, the present research aims to develope isotropic mixtures and to evaluate the effects of boswellic acids formulated in novel nanoemulsion based hydrogel which accomplish significant anti- inflammatory effect on rats using carrageenan-induced rat paw edema model.

\section{Materials and Methods}

\section{Material}

The dry extract of Boswellia serrata (B. Serratta) was gifted by Pharmanza Herbal Pvt. Ltd., Gujarat, India. Coconut oil and clove oil were purchased from 
S.D Fine chemicals, Mumbai, India. Caprylic triglyceride polyethyleneglycol-4 complex (Labrafac $\AA$ ), Diethylene glycol monoethyl ether (Transcutol $\mathrm{P} \AA$ ), Oleoyl macroglycerides EP (Labrafil), Polyglyceryl-6dioleate (Plurol-Oleique $\left.{ }^{\circledR}\right)$, were received as gift samples from Gattefossé (Cedex, France). Sasol Imwitor, Acrysol (Hydrogenated castor oil) were provided as gratis samples from Abitec Corporation, Mumbai, India. Oleic acid, Triacetin and Isopropyl Myristate, (IPM) were procured from SD Fine chemicals, (Mumbai, India) Span 20, Span 60, Tween 80 and Carbopol 940 were purchased from Sigma Aldrich (St. Louis, MO). HPLC grade methanol and phosphoric acid, Acetonitrile were purchased from SD Fine chemicals (Mumbai, India). All other chemicals, buffer solution components and solvents were of analytical grade. Water was obtained from Milli $Q$ water purification system (Millipore, MA).

\section{Excipient Screening Method}

The equilibrium solubility study was performed by adding an excess amount of $B$. serrata extract in $2 \mathrm{~mL}$ of various oils (isopropyl myristate, Labrafac, oleic acid, Labrafil, coconut oil, clove oil, almond oil and Sasol Imwitor), surfactants (Tween 80, Span 60, Span 20 and Acrysol) and cosurfactants (Plurol Oleique and Transcutol P) in $5 \mathrm{~mL}$ capacity stoppered vials separately vortexed using a Cyclo mixer [CM 101, REMI (INDIA)]. The vials were then kept at $25 \pm 0.5^{\circ} \mathrm{C}$ in an orbital shaker (CSI-24 BL, Remi Laboratories and Ahmadabad, India) for $72 \mathrm{~h}$ to reach equilibrium. Following attainment of equilibrium, the supersaturated samples were centrifuged at 2,000 rpm for $15 \mathrm{~min}$ to separate undissolved amount. The obtained supernatants were quantified followed by filtered through a $0.45 \mu \mathrm{m}$ membrane filter (Membrane Technologies, (Mumbai, India) using a HPLC (Shimadzu, Tokyo, Japan).

\section{Construction of Phase Diagrams and Formulation of BAs-loaded NE}

\section{Construction of Pseudo Ternary Phase Diagrams}

On the basis of solubility studies, selected as oil (IPM), surfactant (Tween 80), co-surfactant (Transcutol P) and distilled water were used to construct phase diagram. Surfactant and co-surfactant (Smix) were mixed in different weight ratios (Km 1:1, 2:1, 3:1, 4:1 and 1:2, 1:3). For drawing phase diagrams, ten different combinations of oil and Smix (1:9, 2:8, 3:7, 4:6, 5:5, 6:4, 7:3, 8:2, 9:1 and 1:0) were combined in different glass tubes to cover entire boundaries of three phases precisely formed in the phase diagrams. Water titration method was adopted and samples were examined for transparency and flowability by visual observations. The existence of nanoemulsion zone can be illustrated by constructing pseudo ternary phase diagram using ProSim ternary software.

\section{Formulation of B. Serrata Loaded Nanoemulsion} System

To prepare B. Serrata extract-loaded NE, appropriate amount of oil surfactant and cosurfactant were mixed together in accordance with obtained microemulsion region in the phase diagram and equilibrated with gently vortexing to get the initial concentrate. Then appropriate amount of $B$. serrata extract was dissolved in the oil phase followed by addition Smix to prepare initial preconcentrate. The blend was mixed using high speed homogenizer at 6000RPM upto $10 \mathrm{~min}$. [IKA Pvt. Ltd, Germany] as the extract was resinous in nature. The drop wise addition of water was done to resulting mixture till nanoemulsion was formed.

\section{Characterization of Nanoemulsion}

The morphology and structure of the droplet present in nanoemulsion was analyzed using electron microscope (Tecnai G2 20 TEM, Phillips Holland) operating at $70 \mathrm{kV}$ capable of point-to-point resolution. The differential scanning calorimeter (DSC-6 Perkin Elmer, USA) was used for detecting changes in specific heat capacities of sample in an aluminum crimped pan.

The mean droplet size and zeta potential of the various nanoemulsions was determined by photon correlation spectroscopy using a Zetasizer 1000 HS (Malvern Instruments, Worcestershire, UK). Sample was extemporaneously diluted in Milli-Q water (Millipore Corp., USA) and injected in the apparatus. Each sample was analyzed twice, each analysis consisting of five replicates. The viscosity of the nanoemulsion was determined using Brookfield viscometer LV model (DVIII Ultra Programmable Rheometer) using spindle no. S-61 in triplicate at $25^{\circ} \mathrm{C}$. The refractive index of the prepared nanoemulsion was measured by an Abbe refractometer (Nirma International, Mumbai) to reveal isotropic character of $\mathrm{NE}$ in triplicate at $25^{\circ} \mathrm{C}$. In order to verify the isotropic nature of nanoemulsion, optimized sample was examined using cross-polarized light microscopy (Leica microsystem, Germany) by putting drop of sample between a coverslip and glass slide and then observed under cross-polarized light. The equilibrium surface tension of samples was measured using Surface tensiometer (Force Tensiometer K100, Kruss, Germany).

The $\mathrm{pH}$ values of nanoemulsions were determined using Digital pH meter (Weltronix PM100, EIE, INDIA) in triplicate at $25^{\circ} \mathrm{C}$. Electrical conductivity $(\sigma)$ of the sample was measured using a conductivity meter CDM230 in duplicate to ensure reproducibility. To determine the solubility of BAs in NE, excess B. Serrata was added into $2.5 \mathrm{~mL}$ initial preconcentrate, then $5 \mathrm{~mL}$ water was added and the resulting dispersion was shaken at $25^{\circ} \mathrm{C}$ for $72 \mathrm{~h}$ using an orbital shaker and centrifuged at 10,000 rpm. The supernatant was diluted with methanol appropriately and the content of BAs was determined by HPLC. The formulations were exposed to 
centrifugation (10,000RPM for $20 \mathrm{~min})$ and heatingcooling cycle $\left(-4^{\circ} \mathrm{C}\right.$ and $40^{\circ} \mathrm{C}$ for $48 \mathrm{hrs}$.) to assess thermodynamic stability.

\section{in-vitro Permeation Experiments}

\section{in-vitro Drug Diffusion Study}

in vitro diffusion studies of nanoemulsions were performed using a modified Franz diffusion cell attached with thermostatic water bath at $25^{\circ} \mathrm{C} \pm 1{ }^{\circ} \mathrm{C}$. A dialysis membrane (MWCO: 12,000 Da, Himedia), with a pore size of $0.45 \mu \mathrm{m}$ was used. Each one gm of drug loaded nanoemulsion was placed in the donor compartment. The receiver compartment was filled with phosphate buffer ( $\mathrm{pH}$ 7.4) as dialysis medium at $25^{\circ} \mathrm{C} \pm 1^{\circ} \mathrm{C}$ and $400 \mathrm{rpm}$ on magnetic stirrer. Aliquots were periodically withdrawn at suitable time interval from the receiver compartment through a side tube and analyzed using HPLC.

\section{Ex-Vivo Skin Permeation Study}

The ex vivo skin permeation study was performed using vertical Franz diffusion cell having effective diffusional area $7.03 \mathrm{~cm}^{2}$ and $15 \mathrm{~mL}$ of receiver chamber capacity. Skin samples were prepared by removing subcutaneous fat and connective tissues, washed and examined for integrity. Skin was soaked in the receptor solution for $1 \mathrm{~h}$ before the permeation experiment. Skin sample was then clamped between the donor and the receptor chamber of vertical diffusion cells. Samples were gently placed in the donor chamber and the receptor chamber was filled with PBS (pH 7.4) and thermo stated at $25^{\circ} \mathrm{C} \pm 1^{\circ} \mathrm{C}$. The solution in the receptor chamber was stirred using magnetic stirrer at $100 \mathrm{rpm}$ throughout the experiment. Samples were withdrawn at 0.5, 1, 2, 4, 6 and $8 \mathrm{hr}$., filtered through membrane filter and analyzed for BAs permeant content using HPLC method. Calculation of permeation data was done by plotting the graph of the cumulative amount of drug permeated through the skin $\left[\mathrm{mg} / \mathrm{cm}^{2}\right]$ as a function of time $[\mathrm{t}, \mathrm{h}]$ for formulation. The flux at steady state $\left[\mathrm{J}_{\mathrm{ss}}\right]$ was calculated by dividing the slope of the linear portion of the graph by the area of diffusion cell. The permeability coefficient $\left[\mathrm{K}_{\mathrm{P}}\right]$ and Enhancement ratio was calculated using following Equation 3 and 4 respectively (Shakeel et al., 2007).

Calculation Permeation Data using following Equations 1 and 2:

$$
\begin{aligned}
& K_{p}=\frac{J_{s s}}{C_{0}} \\
& E_{r}=\frac{J_{s s} \text { of formulation }}{J_{s s} \text { of control }}
\end{aligned}
$$

\section{Skin Irritation Study}

The skin irritation test was carried out on Male Sprague Dawley rats weighing 250-300 g. A single dose of $10 \mathrm{mg}$ of the nanoemulsion hydrogel was applied to the left ear of the rat, with the right ear as a control. The development of erythema was monitored for 6 days.

\section{in-vivo Pharmacodynamics Study using Carrageenan-Induced Rat Paw Edema}

Male Sprague Dawley rats weighing (250-300 gm, 12-14 weeks age) were housed in cages with free access to standard diet and water ad libitum, acclimatized to surrounding for one week prior to experiment. The experimental protocol was approved by Institutional Animal Ethics Committee of Faculty of Pharmacy, Dharmsinh Desai University, Nadiad, India [Reg. no/date: No. 1338/c/CPCSEA, 07/04/2010] as per the guidance of CPCSEA, Ministry of Social Justice and Experiment, Government of India with approved Protocol No: DDU/FOP/14/04, dated 19th March 2014. The committee guidelines were followed for the skin permeation, skin irritation and anti-inflammatory studies. The carrageenan-induced hind paw edema method was used to examine anti-inflammatory effect of the optimized nanoemulsion based hydrogel formulation. Rats were fasted for $12 \mathrm{~h}$ prior to the treatment although the water was in access during the fasting period. Male Sprague Dawley rats were randomly allocated to three groups, each containing six animals. Group I $(n=6)$ : Served as Normal control; received plain carbopol gel without drug. Group II $(n=6)$ : Served as standard group; received Standard Piroxicam gel. Group III $(n=6)$ : Served as test group; received nanoemulsion based hydrogel formulation. A total of $0.1 \mathrm{~mL} 1 \%$ $(\mathrm{v} / \mathrm{v})$ suspension of carrageenan in normal saline was injected intradermally into subplantar region of right hind paws of the rats. Each test compound was applied topically $1 \mathrm{~h}$ prior to the injection of carrageenan. The digital vernier caliper [RSK, Mumbai] was used to measure paw width and thickness before and at 1st, 2nd, 3rd, 4th and 5th hour after injection of carrageenan the paw volume was then calculated from width (a) and thickness (b) measurement using the following Equation 3 (Giraldi et al., 1994):

$$
\text { Volume }=\pi \times a^{2} \times b
$$

The percentage inhibition of paw edema was calculated for each group with respect to its vehicletreated control group by using the formula:

$$
\text { Percentage inhibition of edema }=\left(1-V_{t} / V_{c}\right) \times 100
$$

where, $\mathrm{V}_{\mathrm{t}}$ is the inflammatory increase in paw volume in test groups and $V_{c}$ is the inflammatory increase in paw volume in normal control group of rats. Percentage inhibition of edema is proportional to antiinflammatory activity. 


\section{Statistical Analysis}

The statistical significance difference of mean values was assessed using one way ANOVA followed by Dunnet's multiple comparison Test using GraphPad prism 6 software. Statistical probability (p) values less than 0.05 were considered significantly different.

\section{Results and Discussion}

\section{Screening of Oil for $N E$}

The solubility of boswellic acids was determined in different oils (Fig: 1-A.1). It was evident that BAs exhibited highest solubility in isopropyl myristate $\left(192.71 \pm 0.89 \mathrm{mg} 2 \mathrm{~mL}^{-1}\right)$ as polarity of the poorly soluble drugs favors their solubilization in small/medium molar volume oils. Edible oils cannot depicts large microemulsion region due to their rancid nature. IPM was selected for the preparation of nanoemulsions due to its well-known permeationenhancing property and biocompatibility.

\section{Selection of Surfactant Co-Surfactant System}

It is necessary to determine the type and concentration of surfactant as nonionic surfactants known to be less affected by changes in $\mathrm{pH}$ and ionic strength (Azeem et al., 2009). Amongst various surfactants screened, Tween 80 exhibited $(93.8 \pm 1.23 \mathrm{mg}$ $2 \mathrm{~mL}^{-1}$ ) highest solubilization capacity of BAs. Whereas various cosurfactants were screened for solubility as well miscibility with surfactant, Transcutol $\mathrm{P}$ was revealed greater solubilization capacity $\left(101.98 \pm 1.02 \mathrm{mg} 2 \mathrm{~mL}^{-1}\right)$ as well as it forms transparent system $(99.87 \% \mathrm{~T})$ more than Plurol Oleique $(96.78 \% \mathrm{~T})$ with selected surfactant at different $\mathrm{Km}$ values. Transcutol $\mathrm{P}$ also increased permeant partitioning into and solubility within the stratum corneum depicts as permeation enhancer. The result of solubility of BAs in various surfactant and cosurfactants are shown in Fig. 1-A.2. In conclusion, IPM, Tween 80 and Transcutol P were subsequently chosen as the oil phase, surfactant and cosurfactant for the construction of phase diagrams.

\section{Construction of Pseudo Ternary Phase Diagram}

Pseudo ternary phase diagrams were constructed in the absence of BSE to identify nanoemulsion region depicts an important tool to study the phase behavior of nanoemulsion. It can be represented in a triangular format (triangle) which has three coordinates. (1) Oil phase, (2) Surfactant/co-surfactant phase and (3) aqueous phase at fixed weight ratios (Smix ratios 1:1, 2:1, 3:1, $4: 1,1: 2,1: 3)$. The different blend of surfactant and cosurfactants $(\mathrm{Km})$ were chosen for detailed study of the phase diagrams (Azeem et al., 2002). The concentrations of components were recorded in order to complete the pseudoternary phase diagrams and then the contents of oil, Smix and water at appropriate weight ratios were selected based on these results.

An o/w nanoemulsion region was found towards the water-rich apex of the phase diagram. As the surfactant concentration was increased in the Smix ratio 1:1 (Fig. 2a), a higher nanoemulsion region was observed. The probable reasons are reduction of the interfacial tension, increased the fluidity of the interface. Kawakami $\mathrm{K}$ and coworkers reported that greater penetration of the oil phase in the hydrophobic region of the surfactant monomers (Kawakami et al., 2002). From the phase diagrams in Fig. $2 \mathrm{e}$ and $\mathrm{f}$, formulation with $\mathrm{Km} \mathrm{1:2,} \mathrm{1:3} \mathrm{showed} \mathrm{very} \mathrm{narrow} \mathrm{area} \mathrm{of}$ microemulsion formation compared with others.

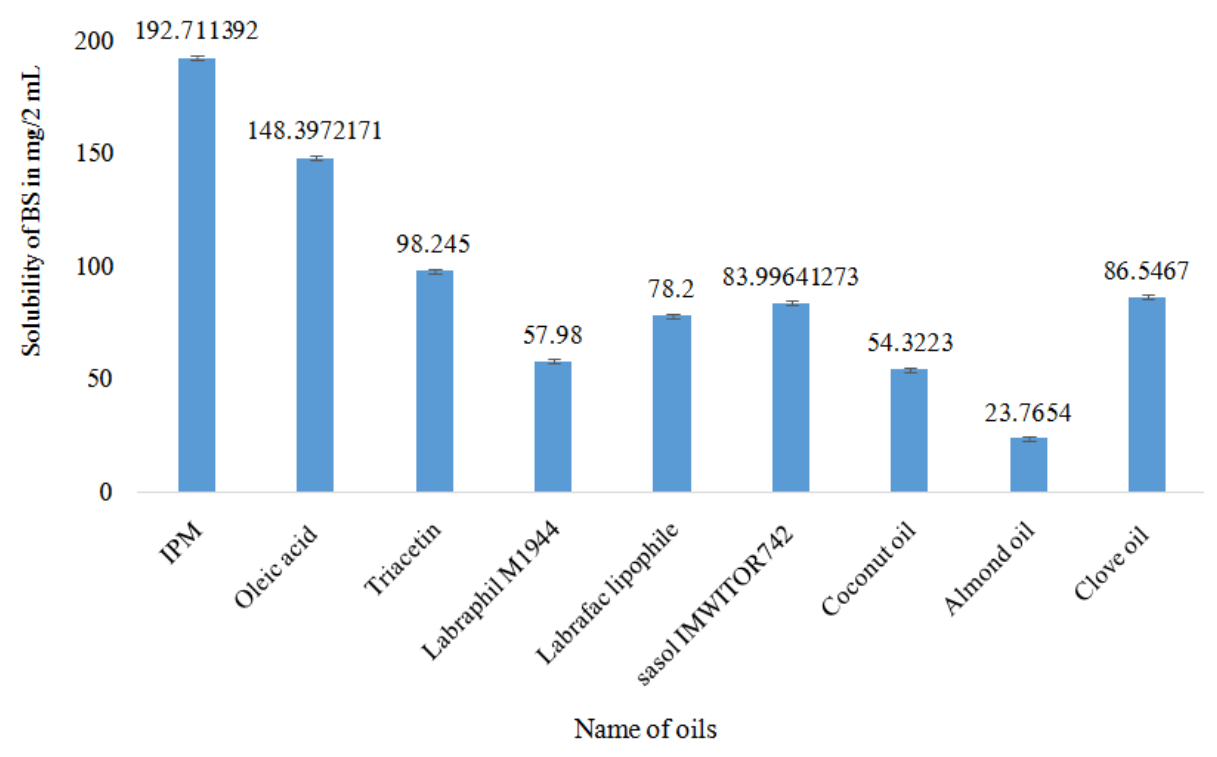

(A) 


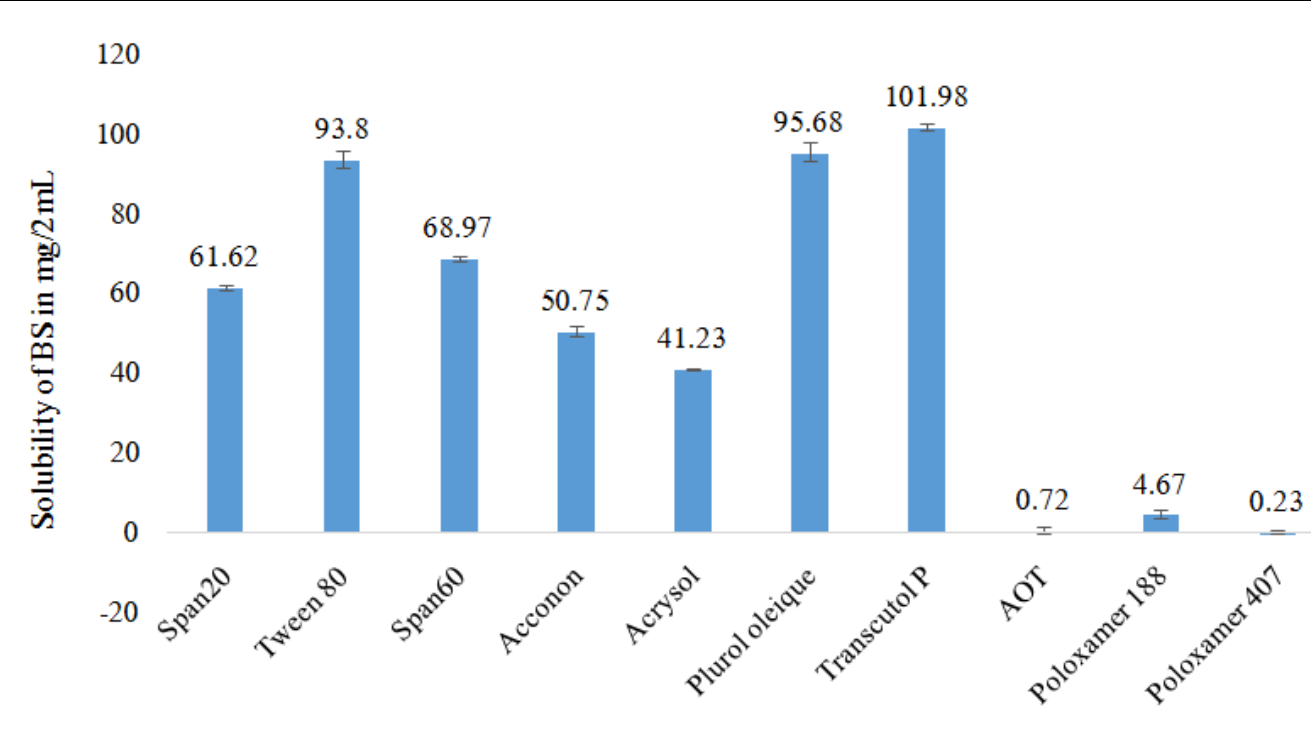

Name of surfactants and cosurfactants

(B)

Fig. 1. (A) Solubility of Boswellic acids in various oils $(n=3)$ (B) Solubility of Boswellic acids in various surfactants and co surfactant $(n=3)$
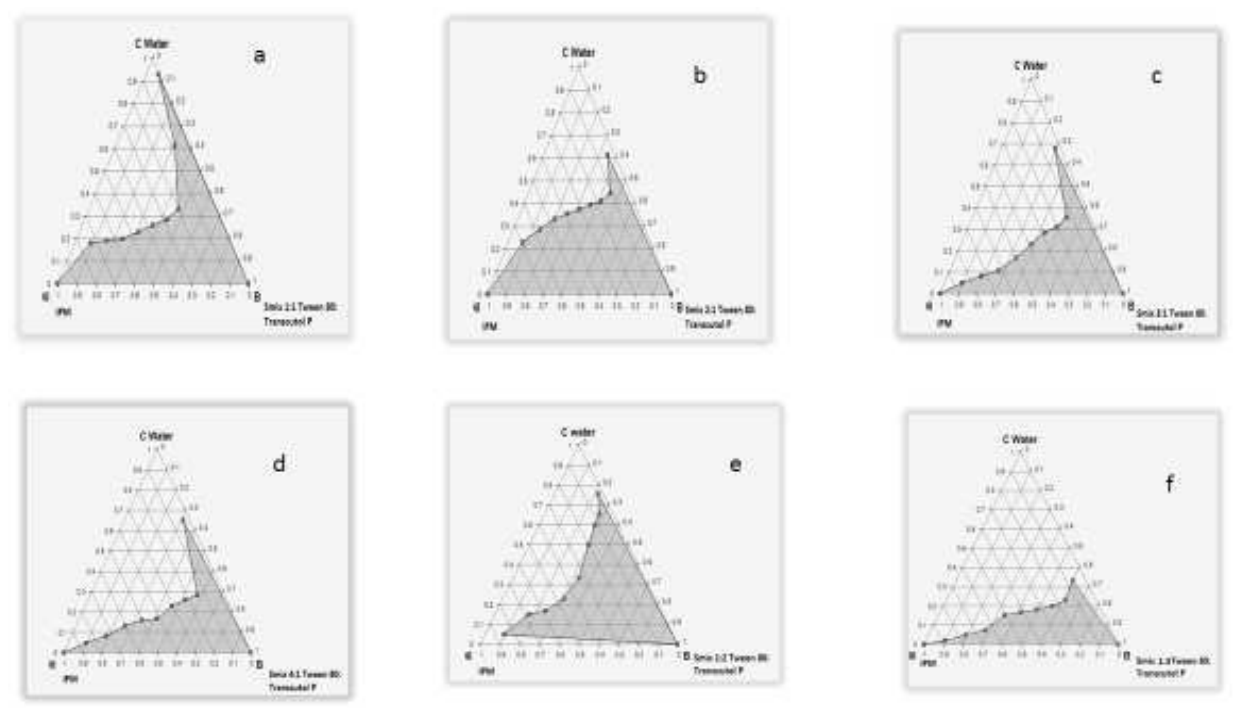

Fig. 2. Pseudo ternary diagrams with Km (a) 1:1, (b) 2:1, (c) 3:1 and (d) 4:1, (e) 1:2 (f)

So, further study in direction of increasing concentration of cosurfactant was not carried out. Phase diagram in Fig. 2a and $\mathrm{c}$ with $\mathrm{Km} \quad 1: 1$ and 3:1 has largest area of microemulsion formation followed by Km 4:1 (Fig. 2d). Beyond this point, there is decrease in the area of NE formation. Various blends were selected from pseudo ternary phase diagrams, the formulations in which the amount of oil phase completely solubilized the drug and which could accommodate the optimum quantity of Smix and distilled water were selected for the development of isotropic mixtures and further used for characterization.

\section{Characterization of Nanoemulsion}

TEM positive image of optimized NE showed spherical droplets without aggregation confirmed the findings of dynamic laser scattering technique (Fig. 4 and 5). DSC was applied to confirm the alteration in physical property of the drug. As specific endothermic peak of untreated drug was found at $104.92^{\circ} \mathrm{C}$ with heat capacity of $-777.75 \mathrm{~mJ}$, while in case of drug loaded nanoemulsion, the specific endothermic peak was found at $78.47^{\circ} \mathrm{C}$ with heat capacity of $-46.16 \mathrm{~mJ}$. 


\begin{tabular}{rllll}
\hline \multicolumn{1}{l}{ Results } & & & & \\
& & Size (r.nm): & \% Intensity & Width (r.nm): \\
Z-Average (r.nm): 11.68 & Peak 1: & 12.20 & 94.7 & 4.197 \\
Pdl: 0.208 & Peak 2: & 1948 & 5.3 & 558.2 \\
Intercept: 0.957 & Peak 3: & 0.000 & 0.0 & 0.000
\end{tabular}

Result quality: Good

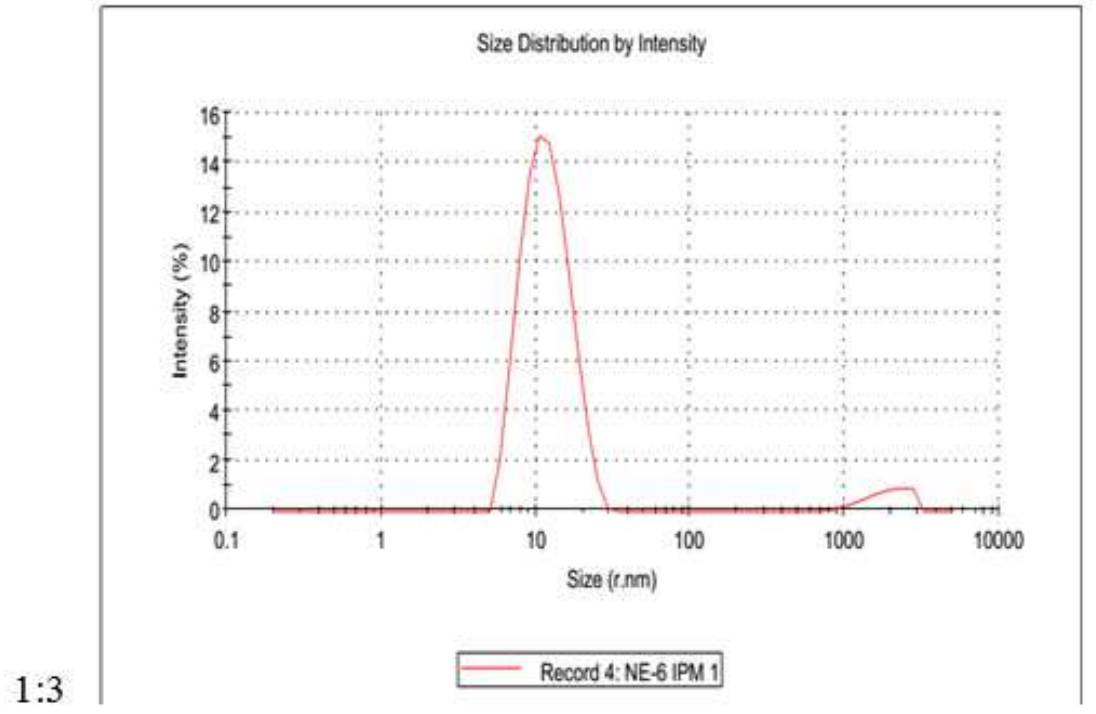

Fig. 3. Droplet size distribution

\section{Results}

Potential $(\mathrm{mV}): 0.226$

Zeta Deviation (mV): 3.65

Conductivity (mS/cm): 0.164

Mean $(\mathrm{mV}) \quad$ Area $(\%) \quad$ Width $(\mathrm{mV})$

$\begin{array}{llll}\text { Peak 1: } & 0.226 & 100.0 & 3.65 \\ \text { Peak 2: } & 0.00 & 0.0 & 0.00 \\ \text { Peak 3: } 0.00 & 0.0 & 0.00\end{array}$

Result quality: See result quality report

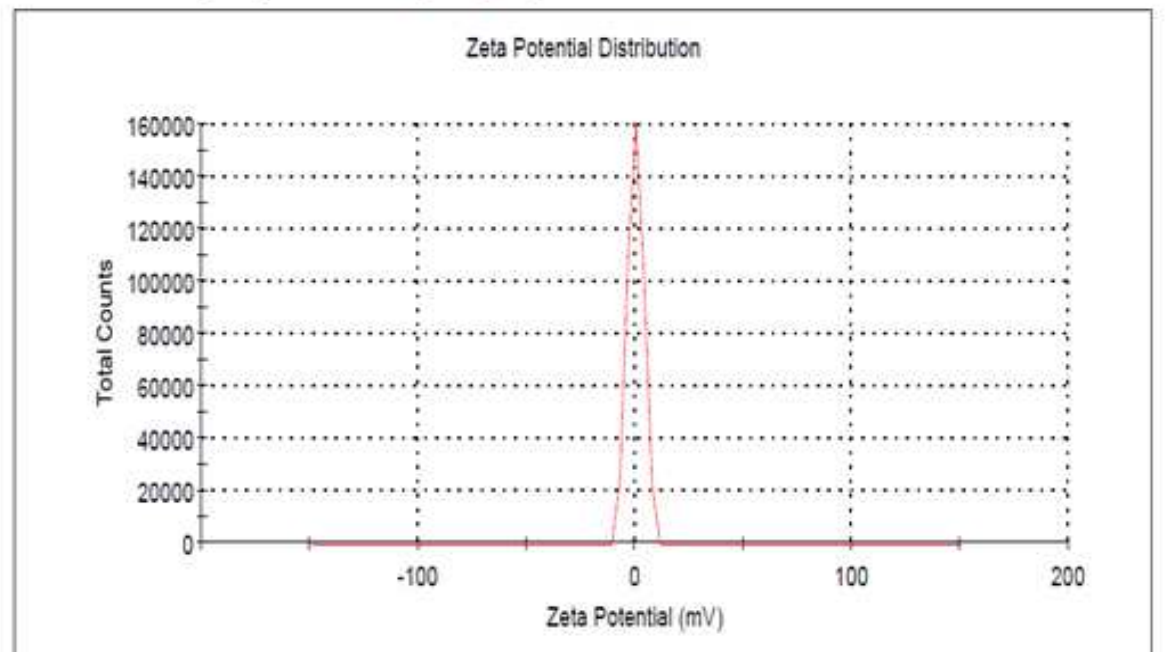

Fig. 4. Zeta potential of nanoemulsion 


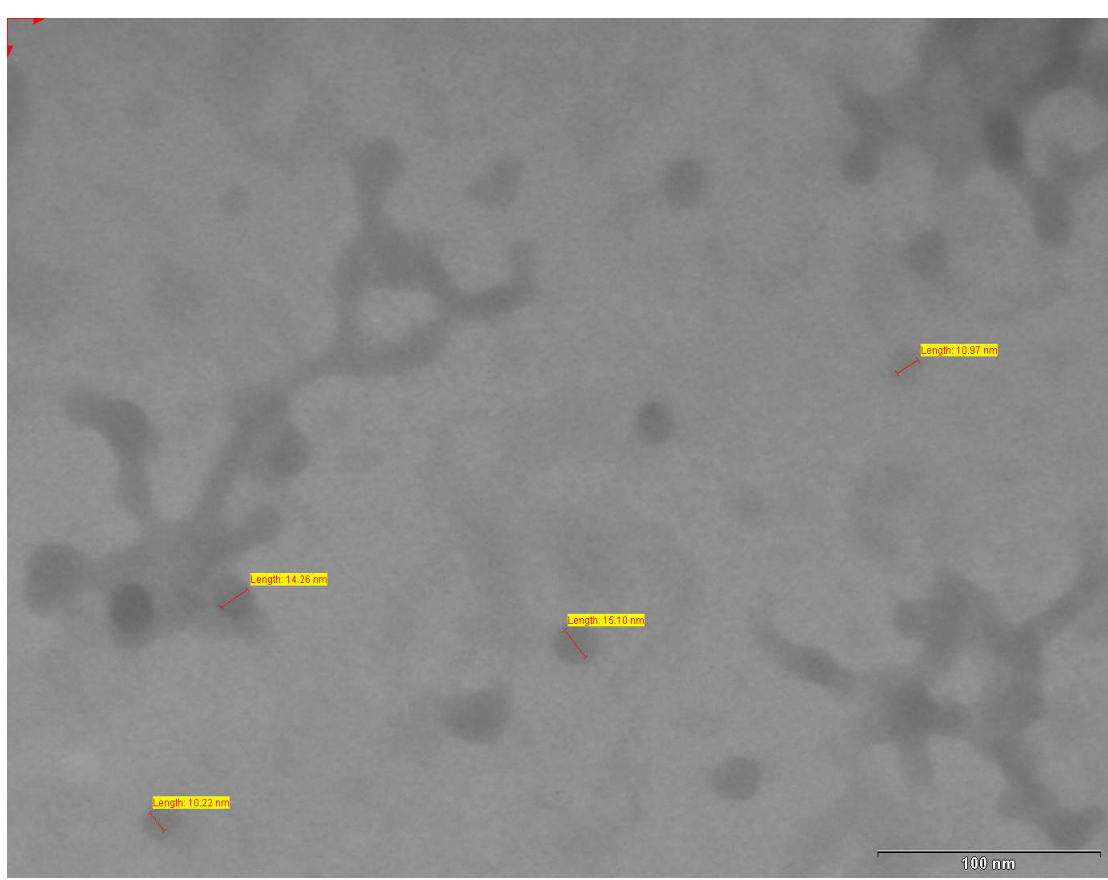

Fig. 5. TEM image of optimized Nanoemulsion formulation at $100 \mathrm{~nm}$ scale
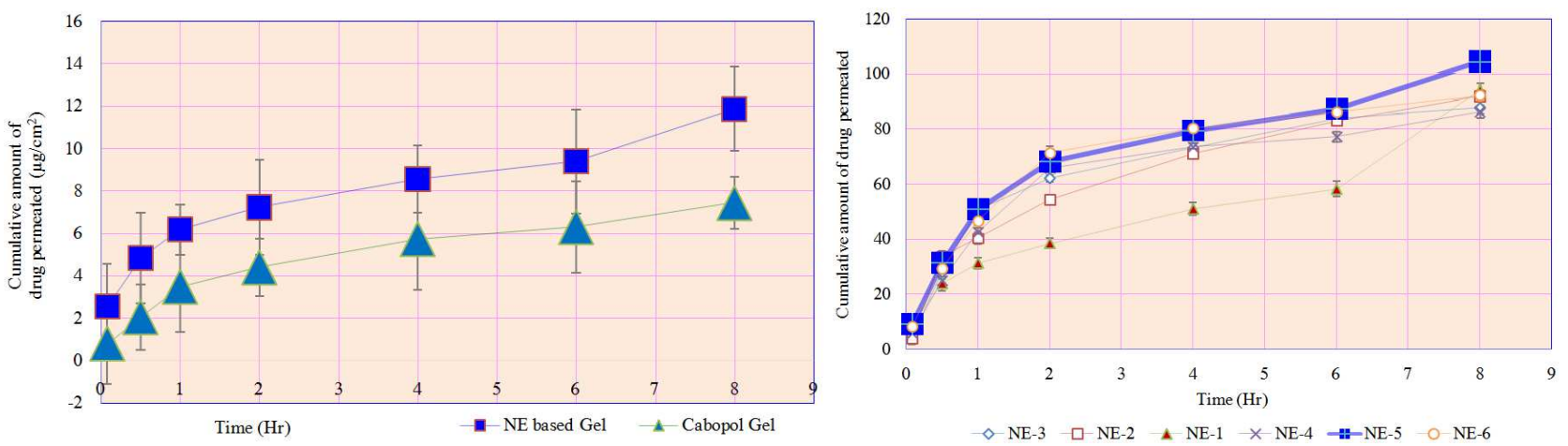

Fig. 6. in vitro diffusion profile of prepared nanoemulsion formulations

The droplet size of prepared formulations were in the range of $11.2 \pm 0.25 \mathrm{~nm}$ to $34.78 \pm 1.25 \mathrm{~nm}$. The low polydispersity values observed for all the formulations, Zeta potential determination of the prepared formulations were in range of $0.226 \pm 0.01 \mathrm{mV}$ (shown in Fig. 3) to $-0.846 \pm 0.40 \mathrm{mV}$. The nanoemulsions consisted of nonionic components, which show relatively neutral charge. This means that absorption is not influenced by charge of membrane in body. The viscosity of nanoemulsions were found in range of $22.3 \pm 1.89$ to $29.1 \pm 2.56 \mathrm{~m}$.Pa.s. As the concentration of oil globules was increased, viscosity of the formulation was increased. The $\mathrm{pH}$ of prepared formulation were found in range of $6.23 \pm 0.02$ to $6.67 \pm 0.08$. The refractive index of prepared formulations were found in the range of $1.28 \pm 0.01$ to $1.99 \pm 0.02$ (Table 1). However little increase in the refractive index was seen in case of formulation having more oil content and less water content, as refractive index of water, i.e., 1.334.

Examination under cross polarizing microscope showed dark field indicating no change in isotropic character. No crystals of the drug were detected that confirmed optical isotropic property of formulation and drug was not in liquid crystalline phase. Percentage transmittance of prepared formulation were found in range of $96.24 \pm 0.087$ to $99.87 \pm 0.036$ indicating transparency and optical isotropic nature of the formulations. The conductance of the formulation increased as water level was increased indicates $\mathrm{O} / \mathrm{W}$ type of nanoemulsion. The 5.5 fold increasing in solubility of BAs found by incorporating in nanoemulsion system. The viscosity of NE based hydrogel were found in range of $74.23 \pm 2.28 \mathrm{mPa}$. S with $6.80 \pm 1.15$ $\mathrm{pH}$ having Spredability $2.35 \mathrm{~cm} . \mathrm{gm} / \mathrm{sec}$ (Table 2). 


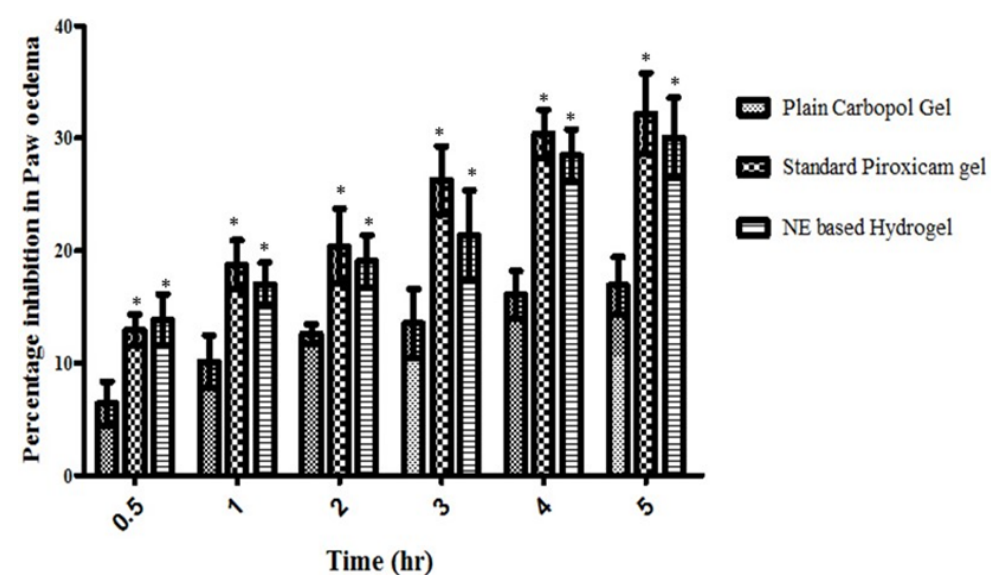

Fig. 7. Time-dependent inhibitory effect of the topical application of NE based Hydrogel of Boswellic acids against carrageenan-induced paw edema in rats. Data are expressed as mean \pm S.E.M. ( $n=6$ /group). Asterisks denote significance levels compared with control values $* \mathrm{p}<0.05$

Table 1. The influence of mixture components on characterization of prepared nanoemulsion $(n=3)$

\begin{tabular}{|c|c|c|c|c|c|c|c|}
\hline Mixtures & $\begin{array}{l}\text { Refractive } \\
\text { Index }\end{array}$ & $\begin{array}{l}\text { Zeta } \\
\text { potential (mv) }\end{array}$ & $\begin{array}{l}\text { Polydispersity } \\
\text { Index }\end{array}$ & $\begin{array}{l}\text { Viscosity } \\
\text { (m Pa .s) }\end{array}$ & $\begin{array}{l}\text { Surface Tension } \\
\text { dyne } / \mathrm{cm} \text {. sec }\end{array}$ & $\mathrm{pH}$ & $\%$ Transmittance \\
\hline$\overline{\mathrm{NE}-1}$ & $1.28 \pm 2.1$ & $-0.54 \pm 0.14$ & 0.205 & $22.3 \pm 3.25$ & $27.68 \pm 0.04$ & $6.61 \pm 0.02$ & $98.12 \pm 0.005$ \\
\hline NE-2 & $1.54 \pm 1.2$ & $-0.08 \pm 0.45$ & 0.890 & $22.1 \pm 2.01$ & $27.06 \pm 0.01$ & $6.35 \pm 0.04$ & $99.25 \pm 0.03$ \\
\hline NE-3 & $1.32 \pm 2.2$ & $-0.34 \pm 0.11$ & 0.761 & $22.8 \pm 1.89$ & $26.04 \pm 0.09$ & $6.49 \pm 0.05$ & $98.14 \pm 0.04$ \\
\hline $\mathrm{NE}-4$ & $1.58 \pm 1.8$ & $-0.32 \pm 0.22$ & 0.305 & $23.3 \pm 0.15$ & $25.74 \pm 0.08$ & $6.23 \pm 0.02$ & $99.47 \pm 0.014$ \\
\hline $\mathrm{NE}-5$ & $1.44 \pm 0.9$ & $0.22 \pm 0.01$ & 0.208 & $23.9 \pm 1.54$ & $25.70 \pm 0.07$ & $6.62 \pm 0.04$ & $99.35 \pm 0.025$ \\
\hline NE-6 & $1.39 \pm 2.6$ & $-0.13 \pm 0.25$ & 0.744 & $22.7 \pm 1.62$ & $25.68 \pm 1.02$ & $6.87 \pm 0.03$ & $99.87 \pm 0.036$ \\
\hline NE-7 & $1.89 \pm 1.4$ & $-0.79 \pm 0.03$ & 0.701 & $25.4 \pm 2.54$ & $31.19 \pm 2.05$ & $6.41 \pm 0.03$ & $96.24 \pm 0.087$ \\
\hline
\end{tabular}

Table 2. Result of $\mathrm{pH}$, Droplet size, Viscosity, Spredability and $\%$ drug content of prepared formulation $(\mathrm{n}=3)$

\begin{tabular}{llllll}
\hline Formulation & $\mathrm{pH}$ & Droplet size $\mathrm{nm}$ & Viscosity m Pa.S & Spredability cm.gm/sec & $\%$ Drug content \\
\hline Optimized NE & $6.62 \pm 0.04$ & $11.2 \pm 1.25$ & $23.9 \pm 1.54$ & $0.12 \pm 1.05$ & $89.12 \% \pm 3.21$ \\
NE based Gel & $6.80 \pm 1.15$ & $54.26 \pm 1.99$ & $74.23 \pm 2.28$ & $2.35 \pm 1.54$ & $92.35 \% \pm 2.98$ \\
Carbopol Gel & $6.95 \pm 1.20$ & $98.74 \pm 2.08$ & $81.25 \pm 3.38$ & $3.54 \pm 1.88$ & $35.65 \% \pm 2.58$ \\
\hline
\end{tabular}

Table 3. Physicochemical parameters of the optimized Nanoformulation during accelerated stability study

\begin{tabular}{lll}
\hline Condition & $40^{\circ} \mathrm{C} / 75 \% \mathrm{RH}$ & $40^{\circ} \mathrm{C} / 75 \% \mathrm{RH}$ \\
\hline Time, months & 3 & 6 \\
Phase separation & No & No \\
\% Transmittance & 99.89 & 99.05 \\
pH & 6.25 & 6.48 \\
Particle size $(\mathrm{nm})$ & 57.13 & 116.9 \\
Zeta potential $(\mathrm{mV})$ & -1.21 & 0.226 \\
\hline
\end{tabular}

\section{in vitro Drug Diffusion Study}

in vitro diffusion studies were performed to ensure drug release prior to ex vivo skin permeation studies. The release profiles of boswellic acids from the different nanoemulsion formulations are illustrated in Fig. 6. The maximum drug diffusion in NE-5 could be due to lowest droplet size and lowest viscosity. Lower viscosity reflecting higher fluidity of oil provided better mobility of drug molecules and consequent continuous controlled drug release form NE 5 mixtures. NE-7 batch was excluded for further study as having poor thermodynamic stability.

\section{Ex-Vivo Permeation Study}

The formulation NBH showed a significant skin permeation profile. The skin permeation profile of NE-
5 was significantly different when compared with that of Carbopol Gel (CG) and nanoemulsion based hydrogel. The significant difference in BAs permeation between liquid nanoemulsion formulations, NE based hydrogel and CG was probably due to the mean size of internal phase droplets, which were significantly smaller in nanoemulsions.

\section{Permeation Data Analysis}

Permeability parameters like steady-state flux (Jss), permeability coefficient (Kp) and enhancement ratio (Er) were higher in nanoemulsions and Nanoemulsion Based Hydrogel $[\mathrm{NBH}]$ formulation as compared with $\mathrm{CG}$. As excipients like IPM and Transcutol P act as permeation enhancers. The result depicts optimized nanoemulsion formulation and NE based hydrogel showed enhanced 
flux (Jss) and permeability coefficient (Kp) as compared to the plain carbopol gel. Optimized NE-5 showed highest flux of $0.306 \pm 0.011 \mu \mathrm{g} / \mathrm{cm}^{2} / \mathrm{h}$., in case of NE based hydrogel showed $0.137 \pm 0.058 \mu \mathrm{g} / \mathrm{cm}^{2} / \mathrm{h}$ while carbopol gel showed $0.0944 \pm 0.019 \mu \mathrm{g} / \mathrm{cm}^{2} / \mathrm{h}$. As compare to carbopol gel, 3.25-fold increase in flux was seen in case of liquid nanoemulsion as well 1.45-fold increase in flux was seen for NE based Hydrogel with highest Enhancement ratio (Er) 4.574 and 1.59 respectively. As the viscosity of nanoemulsion is much lower than NE based hydrogel, the mobility of drugs in nanoemulsion is more facile. Moreover the nanoemulsions affect the stratum corneum structure and reduce the diffusional barrier by acting as a permeation enhancer (Mei et al., 2003; Chang et al., 2005; Changez et al., 2006).

\section{Skin Irritation Study}

The skin irritation test was performed to confirm the safety of the optimized nanoformulation. Van-Abbe and coworkers mentioned that a value between 0 and 9 indicates that the applied formulation is generally not an irritant to human skin (Van Abbe, 1975). The mean skin irritation score for NBH was $2.42 \pm 0.76$. From this results, developed nanoemulsion based hydrogel was safe to be used for topical delivery.

\section{in-vivo Anti-Inflammatory Using Carrageenan Induced Paw Edema}

The Intraplanter injection of carrageenan to hind paw in rats induced an increasing in the paw thickness. These edema had a rapid onset and reached a peak at $5 \mathrm{~h}$ after the challenge. Pretreatment with NE based hydrogel containing BAs, standard Piroxicam gel resulted in 31 and $29 \%$ inhibition in paw edema respectively at $5 \mathrm{~h}$ as compared to plain carbopol gel show significantly suppressed the increase in paw edema after carrageenan injection. Hence as shown in Fig. 7, it is evident that NE based Hydrogel on pretreatment showed significant $(p<0.05)$ percentage inhibition of edema as compared to plain carbopol gel which could be due to enhanced permeation of Boswellic acids through skin. Hence prepared NE based hydrogel containing Boswellic acids produced significant anti-inflammatory effect.

\section{Accelerated Stability Studies}

Developed formulations were found to be stable over period of six month. There is no phase separation, aggregation revealed physically stable formulation, no significant change in droplet size, zeta potential and drug content, $\mathrm{pH}$ was seen during consequent analysis upon exposure to stress conditions of temperature and humidity (Table 3 ).

\section{Conclusion}

The present research successively vouch the therapeutic potential of boswellic acids nanoemulsion for topical delivery with abilities of the boswellic acids permeability from nanoemulsion with its narrow droplet size. The studied nanoemulsion components played key role in permeation enhancing effect. Compared with Carbopol gel, the skin permeation ability of boswellic acids was significantly increased by nanoemulsion. The dose of BSE used to treat inflammation could be decreased due to the high solubility of drug in nanoemulsion inculcates significant anti-inflammatory effect. Present research focused on plant extract loaded formulation development of a novel nanoemulsion based drug delivery system which may furnish important initiative and facet by enhancing bioavailability of herbal drugs.

\section{Funding Information}

The PhD research work was supported by Research grant (GUJCOST/MRP/362) from GUJCOST, Gandhinagar, Gujarat, India. Author is thankful to Dr. Atindra Shukla-Shah Schulman Centre for Surface science and Nanotechnology, Dharmsinh Desai University, Nadiad, Gujarat, India for providing research facilities. Also would like to acknowledge Gattefosse and Abitec Corporation, India for providing the gift samples of Lipid excipients. Author has also expressed thanks to Vaishali Thakkar, Kalpana Patel, Lalji Baldania and Mihir Parikh, Prakash, Dipenti for kind support during research and computation work.

\section{Author's Contributions}

Mukesh Gohel: Planning of Research Work for Formulation Development, Guiding throughout the work, Data treatment etc. Reviewing of manuscript and contribute a lot in manuscript preparation.

Tejal Soni: Planning of Research Work for Formulation Development, Characterization of formulation at research centre, Guiding throughout the work, Reviewing of manuscript etc.

Lal Hingorani: Provided scientific guidance and technical support to at Pharmanza Herbal Industry for plant extract related experimental work, chromatographic study etc.

Nayan Patel: Contributed extensively in Animal Experiments from Animal protocol preparation to the commencement of Animal experiments at animal house for anti-inflammatory study.

\section{Ethics}

This article is original and contains unpublished material. The corresponding author confirms that all of the other authors have read and approved the manuscript and no ethical issues involved. 


\section{References}

Ajazuddin, S.S., 2010. Applications of novel drug delivery system for herbal formulations. Fitoterapia, 81: 680-689. DOI: 10.1016/j.fitote.2010.05.001

Azeem, A., M. Rizwan, F.J. Ahmad, Z. Iqbal and R.K. Khar et al., 2009. Nanoemulsion components screening and selection: A technical note. AAPS PharmSciTech, 10: 69-76.

DOI: $10.1208 / \mathrm{S} 12249-008-9178-X$

Chang, X.L., H.B. Chen, X.Z. Zhao, Z.H. Gao and H.B. $\mathrm{Xu}, 2005$. High-performance liquid chromatography determination of triptolide in vitro permeation studies. Anal. Chim. Acta, 534: 215-221. DOI: $10.1016 /$ j.aca.2004.11.036

Changez, M., M. Varshney, J. Chander and A.K. Dinda, 2006. Effect of the composition of lecithin/npropanol/isopropyl myristate/water microemulsions on barrier properties of mice skin for transdermal permeation of tetracaine hydrochloride: in vitro. Colloids Surf. B: Biointerf., 50: 18-25. DOI: $10.1016 /$ j.colsurfb.2006.03.018

Gerbeth, K., J. Meins, S. Kirste, F. Momm and M. Schubert-Zsilavecz et al., 2011. Determination of major boswellic acids in plasma by high-pressure liquid chromatography/mass spectrometry. J. Pharm. Biomed. Anal., 56: 998-1005. DOI: 10.1016/j.jpba.2011.07.026

Giraldi, T., L. Perissin, S. Zorzet and V. Rapozzi, 1994. Stress, melatonin and tumor progression in mice. N.Y. Ann. Acad. Sci., 719: 526-536.

DOI: $10.1111 /$ j.1749-6632.1994.tb56856.x
Goel, A., F.J. Ahmad, R.M. Singh and G.N. Singh, 2010. 3-Acetyl-11-keto-beta-boswellic acid loadedpolymeric nanomicelles for topical antiinflammatory and anti-arthritic activity. J. Pharm. Pharmacol., 62: 273-278. DOI: $10.1211 /$ jpp.62.02.0016

Kawakami, K., T. Yoshikawa, Y. Moroto, E. Kanaoka and K. Takahashi et al., 2002. Microemulsion formulation for enhanced absorption of poorly soluble drugs: I. Prescription design. J. Control Release, 81: 65-74. DOI: $10.1016 / \mathrm{S} 0168-3659(02) 00049-4$

Mayank, C., M. Kumar, A. Sinhal and A. Saif, 2011. Recent development in novel drug delivery systems of herbal drugs. Int. J. Green Pharm., 5: 87-94. DOI: $10.4103 / 0973-8258.85155$

Mei, Z.N., H. Chen, T. Weng, Y.J. Yang and X.L. Yang, 2003. Solid lipid nanoparticle and microemulsion for topical delivery of triptolide. Eur. J. Pharm. Biopharm., 56: 189-196. DOI: 10.1016/S0939-6411(03)00067-5

Shakeel, F., S. Baboota, A. Ahuja, J. Ali and M. Aqil et al., 2007. Nanoemulsions as vehicles for transdermal delivery of aceclofenac. AAPS PharmSciTech, 8: 191-199. DOI: 10.1208/PT0804104

Uthaman, S., K.S. Snima, M. Annapoorna, K.C. Ravindranath and V. Shanti, 2012. Novel Boswellic acids Nanoparticles induces cell death in Prostate cancer cells. J. Natural Products, 5: 100-108.

Van Abbe, N., 1975. Exaggerated exposure in topical irritancy and sensitization testing. J. Soc. Cosmet. Chem., 26: 173-187. 